nifikant stärker angestiegen als unter der fortgeführten Alendronatbehandlung $(+1,05 \%)$. Knochendichtemessungen an LWS, Femur, Trochanter und distalem Radius ergaben ebenfalls eine stärkere Zunahme unter dem RANK-Ligand-Inhibitor.

Hofbauer wies darauf hin, dass mit der nur halbjährlichen Subkutan-Injektion von
Denosumab gleichzeitig eine sehr einfache und patientenfreundliche Anwendung möglich ist.

- Dr. med. Andreas Fischer

Quelle: Pressekonferenz, Osteologie-Kongress, Fürth, 23. März 2011 (Veranstalter: Amgen und GlaxoSmithKline)

Neu von Sanofi-Aventis

\title{
Blutzucker-Messgeräte mit Schnittstelle zu iPod touch und iPhone
}

- Der Diabetes-Spezialist Sanofi-Aventis erweitert sein Angebot um innovative Blutzuckermessgeräte. Bei dem BGStar ${ }^{\circledR}$ handelt es sich um ein kompaktes und extrem anwenderfreundliches Messgerät. Griffige Tastatur, großes Display mit Beleuchtung sowie zahlreiche praktische Funktionen zeichnen das Gerät aus. Dazu zählen Warnsignale bei Über- oder Unterzuckerungen, programmierbare Alarme, die an die BZ-Messung erinnern, sowie positive Feedbackfunktionen bei Messungen im Normbereich.

Technisch ist das Gerät auf dem neuesten Stand. Es verfügt über eine hohe Messgenauigkeit aufgrund dynamischer Elektrochemie-Technologie. Nur 0,5 Mikroliter Blut sind notwendig, das Ergebnis wird in nur sechs Sekunden angezeigt. Knapp 2000 Messwerte werden gespeichert. Eine Diabetes-Management-Software ermöglicht zahlreiche Funktionen, die sowohl dem Patienten (Modus „Zuhause“) als auch dem Arzt (Modus "Professional") eine Dokumentation und Überwachung der Stoffwechseleinstellung ermöglichen.
Noch mehr im Trend liegt das kleinere BZ-Messgerät iBGStar ${ }^{\circledR}$, das über den DockConnector mit iPhone oder iPod touch verbunden werden kann. Das Gerät arbeitet in Verbindung mit einer Diabetes-Manager-App.,

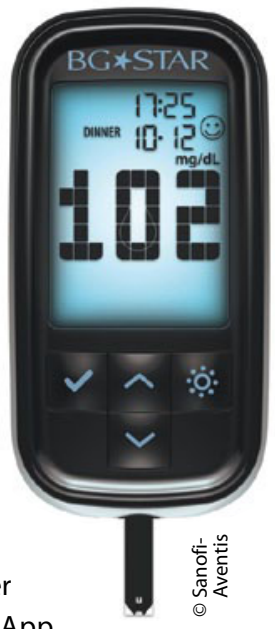
die alle Funktionen des Geräts auf den Touchscreens der Apple-Geräte sichtbar macht. Daten können per EMail direkt versendet werden.

Sanofi-Aventis bietet die BZ-Messgeräte zusammen mit zahlreichen unterstützenden Dienstleistungen an. Dazu gehören eine 24-Stunden-Telefon-Hotline, Broschüren, Webseiten und ein Reklamations- und Austausch-Service. Die Geräte sind seit diesem Monat im Handel (www.bgstar.de).

- $\mathrm{DE}$

\section{Kurz notiert}

Helicobacter eradizieren: einfacher mit Kombipackung Für den Therapieerfolg bei der H.-pylori-Eradikation ist die Compliance der Patienten entscheidend. Erleichtert wird sie durch die neue Kombinationspackung Omep ${ }^{\circledR}$ plus Amoxicillin und Clarithromycin für die siebentägige Therapie. In jedem der Blister ist die entsprechende Morgenund Abenddosis für einen Behandlungstag enthalten. Mit Bildsymbolen auf dem Blister wird die richtige Einnahme sichergestellt. Auf der Internetseite www.ompep-plus.de sind Einnahmehinweise u. a. in Deutsch, Englisch, Türkisch, Polnisch abrufbar (Zugang für Fachkreise: Username „VIP“, Passwort "Arzt"). Hexal AG 